\title{
The effect of goal box delay and separate delay box delay on pattern running'
}

JAMES A. HORN and PATRICK E. CAMPBELL, Southern Methodist University, Dallas, Tex. 75222

Twenty-two rats were trained in a runway under a single-alternation, partially delayed reward schedule. Delay occurred in the goal box or in a separate distinctive delay chamber. The duration of delay was either 20 or $25 \mathrm{sec}$ on all odd numbered trials in a six-trial-a-day schedule. Ss learned to pattern respond, i.e., run rapidly on immediately rewarded trials and slowly on delayed trials, but only when the delay occurred in the separate delay chamber. The duration of delay had no effect on patterning. The results clarify an apparent contradiction in previously reported research and are discussed in terms of the modified aftereffects hypothesis.

Pattern running refers to the tendency of rats to run rapidly on trials to a large incentive and slowly on trials to a smaller incentive provided that the two types of trials occur in a discriminable sequence.
Pattern responding is a well established phenomenon when rewarded and nonrewarded trials occur in single alternation (SA) schedule (Capaldi, 1967). When patterning depends on a discrimination between delayed (D) and immediate (I) trials, however, the data are not in complete agreement. Cogan \& Capaldi (1961) reported patterning in a SA partial reward schedule, but failed to find patterning in a SA $20-\mathrm{sec}$ partial delayed schedule, even after as many as 200 training trials. Burt \& Wike (1963) replicated the Cogan and Capaldi finding in that patterning did not occur when delay confinement duration was at $20 \mathrm{sec}$, but they did report evidence for patterning when delay confinement was increased to 60 or 100 sec. These data led Capaldi (1967) to conclude that the aftereffects of a 20-sec delayed reward were not sufficiently different from the aftereffects of an immediate reward to support a discrimination and, therefore, patterning in a SA situation.

Campbell (1969) reported the results of a SA partial delayed study in which patterning

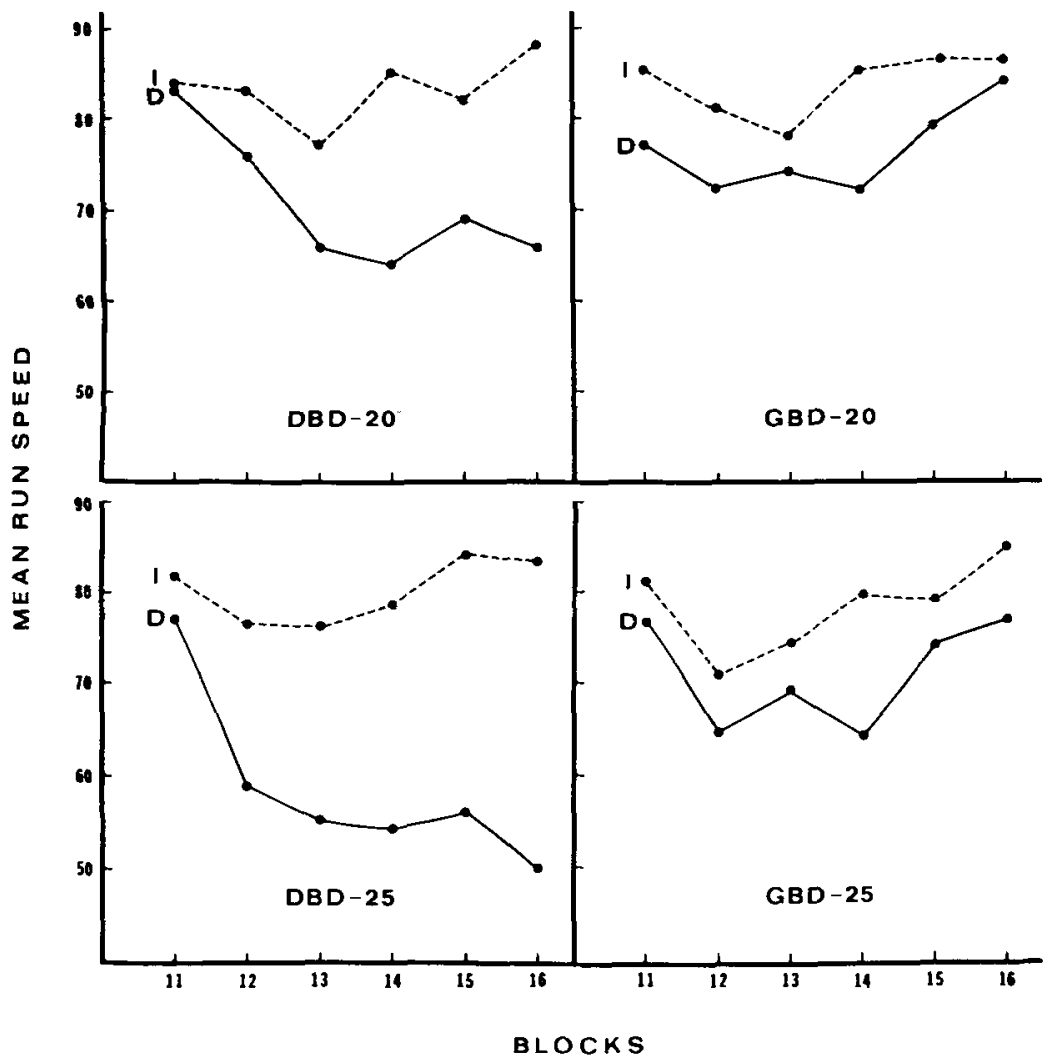

terning occurred rather substantially with delay confinement at $20 \mathrm{sec}$. The Campbell study, however, has several procedural differences from the Cogan and Capaldi and the Burt and Wike experiments. The latter two studies were identical in most respects, including the use of 10 trials a day with delay occurring in the goal box. Campbell used only two daily trials and delay occurred in a separate distinctive delay chamber located immediately before the goal box. Either of these differences could conceivably account for the discrepancy in the results.

The purpose of the present experiment was to determine if pattern responding would occur if a 20 -sec delay occurred in a separate delay chamber, as the Campbell study suggests, and would fail to occur if the delay occurred in the goal box, following the Cogan and Capaldi and the Burt and Wike findings.

\section{SUBJECTS}

The Ss were 22 naive male albino rats, approximately 75 days old when received from the Holtzman Company. Ss were housed in individual cages and maintained at $85 \%$ of free-feeding body weight. Water was continually available in the home cages.

$$
\text { APPARATUS }
$$

The apparatus, a clear plastic Hunter small animal runway, was $57.5 \mathrm{in}$. long, $3.25 \mathrm{in}$. wide, and $5 \mathrm{in}$. high. The entire length was covered with a removable plastic top, and the floor consisted of .13-in. grids spaced .5 in. apart. The initial and final 12-in. sections served as start and goal boxes and were separated from the remainder of the runway by automatically controlled guillotine doors. A 12-in. section immediately preceding the goal box served as the delay chamber and was also separated by an automatic door. The sides of the delay chamber were covered with white construction paper and the remainder of the runway was lined with black paper for contrast. Photoelectric relays were wired to control a Lehigh Valley printout counter which recorded response times for the first 24-in. section of the runway. A second photocell was located $.5 \mathrm{in}$. in front of the goal cup and served to activate delay timers or the pellet dispenser according to the appropriate experimental condition. All timing, response recording, operation of the doors, and pellet delivery were totally automated, using standard relay-base research equipment.

\section{PROCEDURE}

Upon receipt at the laboratory, Ss were given unlimited access to food and water for 15 days. During the final 5 days of free

Fig. 1. Mean response speeds plotted for $D$ and I trial separately for each condition over the last 12 days of training. 
feeding, each $S$ was weighed once a day and its median weight was taken as an estimate of ad lib body weight. Ss were then reduced and maintained at $85 \%$ of this ad lib estimate by make-up feeding given once each day. During the final 3 days allotted to stabilizing Ss' body weights, they were magazine trained to approach and consume .045-g pellets in a Lafayette operant conditioning chamber. A pellet dispenser and goal cup from one of these chambers were subsequently mounted in the goal box of the runway. After completion of the magazine training, Ss were given 1 day of runway exploration and 12 immediately reinforced running trials at four trials a day. On these and all subsequent reinforced trials, reward consisted of five $.045-\mathrm{g}$ Noyes pellets delivered at a rate of one pellet a second, contingent on S's breaking the final photobeam. The Ss were allowed $15 \mathrm{sec}$ to consume the reward.

Following the above pretraining, all Ss were given six rewarded trials a day with a 20-sec delay occurring on all odd numbered trials. A random one-half of the Ss received delay in the goal box (GBD) and the remaining $S s$ were delayed in the separate delay box (DBD). The Ss were run in squads containing equal numbers of $S$ from each delay condition. The squads were handled from unpainted individual handling cages and run in a random order each day. Ss were fed at least $15 \mathrm{~min}$ after the completion of training. After 20 days of this treatment, no suggestion of patterning had occurred, so one $S$ (chosen randomly) from each group was discarded and one-half of the Ss in each group were shifted to a $25-\mathrm{sec}$ delay confinement and the remaining Ss continued to receive $20-\mathrm{sec}$ delays. Training continued for 12 additional days. The intertrial interval was kept at a constant $20 \mathrm{sec}$ throughout the experiment.

\section{RESULTS}

Response times were separated according to $\mathrm{D}$ and $\mathrm{I}$ trials, converted to speed scores (100/time), and averaged over blocks of 2 days. Figure 1 presents $D$ and I trial speeds for the last 12 days of training, plotted separately for each acquisition condition. Inspection of the figure suggests that pattern responding developed in the DBD Ss under each delay confinement condition but did not occur in the GBD Ss, irrespective of delay duration. An analysis of variance performed on the final trial block confirmed these suggestions. D vs I trials (pattern) was significant as a main effect $(F=27.65$, $\mathrm{df}=1 / 16, \mathrm{p}<.(01)$, but also showed a significant interaction with the location of delay $(F=17.13, \quad d f=1 / 16, \quad p<.001)$. Simple main effects, using the compromise error term suggested by Winer (1962) revealed significant pattern effects for $D B D$ $(\mathrm{F}=81.57, \mathrm{df}=1 / 16, \mathrm{p}<.001)$, but not for $G B D(F=1.14, d f=1 / 16, p>.05)$ Furthermore, pattern running was exclusively associated with slower $D$ trial speeds for $\mathrm{DBD}(\mathrm{F}=27.36, \mathrm{df}=1 / 16$, $\mathbf{p}<.001)$ and not with differences in I trial speeds $(F<1.0)$. A nonsignificant Pattern by Delay Duration interaction $(\mathrm{F}<1.0)$ suggested equal patterning at 20. or $25 . \mathrm{sec}$ delays.

\section{DISCUSSION}

These results confirm the findings of Campbell (1969) that pattern responding can occur with delays as short as $20 \mathrm{sec}$. The reason for the failures to find evidence for patterning at 20-sec delay in the Cogan and Capaldi and Burt and Wike experiments is now apparent. Both of their experiments confined the $S s$ in the goal box (GBD) throughout the delay interval. On the basis of the present data, 20-sec GBD would not be expected to support patterning, while $20-$ sec DBD would result in pattern responding.

These results can be incorporated within Capaldi's (1967) sequential trial theory by assuming that the aftereffects of DBD and GBD of the same duration occasion different stimulus aftereffects. These stimuli are conditioned equally to the instrumental response on I trials. This suggestion, of course, is supported by equal I-trial speeds (assumed to reflect the degree of habit conditioned at the two delay af tereffects) in the present experiment. Following this reasoning, however, the delay aftereffects are assumed to be different in their degree of similarity to the aftereffects of immediate reward. The present results dictate the assumption that the aftereffects of GBD are more similar to those of immediate reward than the aftereffects of DBD. Again, this assumption is supported by slower D trial speeds for DBD Ss (DBD is assumed to supply less generalized habit to immediate reward aftereffects than GBD due to less similarity).

An alternative hypothesis, which may account for the functional difference in the effects of the two types of delay, is that GBD could be expected to result in delayed primary reinforcement but immediate secondary reinforcement since contact with the stimuli in the goal box occurs without delay. If this actually occurs, some degree of habit would be conditioned directly to the aftereffects of immediate reward and would summate with the habit generalized from GBD stimuli. The instrumental response would then have both sources of support on D trials and a rapid response would be expected. DBD, on the other hand, is assumed to minimize secondary reinforcement since the delay chamber itself is never associated with reward. Performance on D trials for DBD Ss would be supported only by habit generalized to immediate reward af tereffects and relatively slow responding would be expected. This secondary reinforcement hypothesis does not necessitate the assumption that DBD and GBD result in different aftereffects. Fortunately, an experimental design suggests itself that allows for a test of this assumption. If separate groups of Ss are trained under irregular schedules of partial DBD and GBD, a number of I-I transitions will occur in each group so that, irrespective of secondary reinforcement, asymptotic habits at the I trial aftereffects will develop under each schedule. Any differences in resistance to extinction following such training could be taken as evidence for different aftereffects resulting from the two types of delay. An experiment along these lines is currently being conducted in our laboratory.

\section{REFERENCES}

BURT D. E \& WIKE, E. L.Effects of alternating partial reinforcement and alternating delay of reinforcement on a runway response. Psychological Reports, 1963, 13,439-443.

CAMPBELL, P. E. Sequences of delayed reward and extinction confinement: Effects on pat tern running and extinction performance. Psychonomic Science, $1969,1,34-35$.

CAPALDI, E. J. A sequential hypothesis of instrumental learning. In K. W. Spence and J. T. Spence (Eds.), The psychology of learning and motivation. Vol. 1. New York: Academic Press, 1967. Pp. 67-156.

COGAN, D., \& CAPALDI, E. J. Relative effects of delayed reinforcement and partial reinforcement on acquisition and extinction. Psychological Reports, 1961, 9, 7-13.

WINER, B. J. Statistical principles in experimental design. New York: McGraw-Hill, 1962.

$$
\text { NOTE }
$$

1. This research was partially supported by Grant 8835 from the National Science Foundation to Southern Methodist University. The study was conducted according to the APA statement of "Principles for the care and use of animals," June $26,1968$. 